\title{
Underwater Target characterization and laser imaging system
}

\author{
Walid Gomaa ${ }^{1}$, Ashraf F. El-Sherif ${ }^{2}$, Yasser H. El-Sharkawy ${ }^{3}$
}

Eng. Physics Dept. ${ }^{1,2}$, Biomedical Eng. Dept. ${ }^{3}$, MTC University, Cairo, Egypt.

\begin{abstract}
The conventional method used to detect an underwater target is by sending and receiving some form of acoustic energy. However, acoustic systems have limitations in the range resolution and accuracy. while, the potential benefits of a laser-based underwater target detection include high-directionality, high response, and high precise range accuracy. Lasers operating in the blue-green region of the light spectrum have potential applications in the area of detection and ranging of submersible targets due to minimum attenuation through water and maximum laser reflection of estimated target to provide a long range of detection. The laser echoes from different targets (metal, plastic and wood) were detected using high resolution CCD camera and digital image processing and pattern recognition techniques were applied to detect and discriminate the echoes from these targets by background subtraction and edge detection techniques. As a conclusion, in this paper we present a potential high response laser imaging system to detect and discriminate small size underwater targets.
\end{abstract}

Keywords: Blue-green lasers, target detection, laser imaging system, and edge detection.

\section{INTRODUCTION:}

Water is one of the most important molecules on Earth, which makes our planet different from other known celestial bodies. Water covers about $70 \%$ of the surface of Earth, mostly as saltwater in the ocean $(97 \%)$. Some is fresh water in rivers and lakes; some is in the form of ice or vapor [1].

Underwater range finders have historically used acoustic radiation due to their long range propagation characteristics. However, acoustic systems have limitations in the range resolution and accuracy they can provide under certain conditions [2]. The distance meters utilizing acoustic waves use frequencies in the ultrasonic range from 20 $\mathrm{kHz}$ to some megahertz. The advantages of ultrasonic methods are relatively low frequencies, which are easy to handle with electronics, simple and cheap structure of the device as well as the capability to measure to metal, mirror, and glass surfaces, which are difficult for optical meters. The main disadvantage is the large divergence of the beam (the beam width being some tens of degrees) which apply limitations in the range resolution and accuracy, slowness, multiple reflections and large attenuation in long 
distances. The ultrasound methods are most suitable in short distances (some tens of meters at the maximum) [3]. Optical techniques are currently being investigated due to the potential advantages that lasers provide, including high-directionality, high response, high-precision, and high range accuracy [4].

\section{WATER PROPERTIES AND LASER SOURCE SELECTION:}

The attenuation spectrum of liquid water has been intensively studied, its structure is very complicated since its molecules are connected by hydrogen bonds; the structure of liquid water gives rise to several anomalies when compared to other liquids [5].

The bulk optical properties of water are conveniently divided into two mutually exclusive classes: inherent and apparent. Inherent optical properties (IOP's) are those properties that depend only upon the medium, and therefore are independent of the ambient light field within the medium. The two fundamental IOP's are the absorption coefficient and the volume scattering function. Other IOP's include the index of refraction, the beam attenuation coefficient and the single-scattering albedo. Apparent optical properties (AOP's) are those properties that depend both on the medium (the IOP's) and on the geometric (directional) structure of the ambient light field, and that display enough regular features and stability to be useful descriptors of the water body. Commonly used AOP's are the irradiance reflectance, the average cosines, and the various diffuse attenuation coefficients [6].

Determination of the spectral beam attenuation $c(\lambda)$ for natural waters is a difficult task for several reasons. First, water itself absorbs only weakly at near-UV and blue wavelengths, so that very sensitive instruments are required. More importantly, scattering is never negligible, so that careful consideration must be made of the possible aliasing of the absorption measurements by scattering effects. In pure water at wavelengths of $\lambda=370$ to $450 \mathrm{~nm}$, molecular scattering provides $20-25 \%$ of the total spectral beam attenuation.

$$
c(\lambda)=a(\lambda)+b(\lambda)
$$

where $a(\lambda)$ is the spectral absorption coefficient and $b(\lambda)$ is the spectral scattering coefficient.

The attenuation coefficient $c(\lambda)$ with unit $\mathrm{m}-1$ of the liquid water for electromagnetic radiation, at a particular wavelength is defined as in $I=I_{0} e^{-c(\lambda) L}$ where $I$ is the 
transmitted intensity of light, I0 is the incident intensity of the light and L is the path length [1].

In studies of electromagnetic wave propagation at the level of Maxwell's equations, it is convenient to specify the optical properties of the medium via the electrical permittivity $\varepsilon$, the magnetic permeability $\mu$, and the electrical conductivity $\sigma$. The effects of $\varepsilon, \mu$ and $\sigma$ on electromagnetic plane-wave propagation are compactly summarized in terms of the complex index of refraction, $\mathrm{m}=\mathrm{n}+\mathrm{ik}$, The real part $\mathrm{n}$ of $\mathrm{m}$ is usually called "the index of refraction"; the imaginary part of the index of refraction $\mathrm{k}$ is electrodynamic absorption coefficient, Although $\mathrm{n}$ and $\mathrm{k}$ are collectively called the optical constants of water, they depend strongly on wavelength. The explicit dependence of $\mathrm{m}$ on $\varepsilon, \mu$ and $\sigma$ is given by: [7]

$$
\begin{gathered}
\mathrm{m}^{2}=\mu \varepsilon \mathrm{m}^{2}-\mathrm{i} \frac{2 \pi \mu \sigma \mathrm{c}^{2}}{\mathrm{v}} \\
\mathrm{m}^{2}=(\mathrm{n}-\mathrm{ik})^{2}=\mathrm{n}^{2}-\mathrm{k}^{2}-\mathrm{i} 2 \mathrm{nk}
\end{gathered}
$$

The optical constants are convenient because they are directly related to the scattering and absorbing properties of water. The spectral absorption coefficient $a(\lambda)$ is related to $\mathrm{k}(\lambda)$ by :

$$
\mathrm{a}(\lambda)=\frac{4 \pi \mathrm{k}(\lambda)}{\lambda}
$$

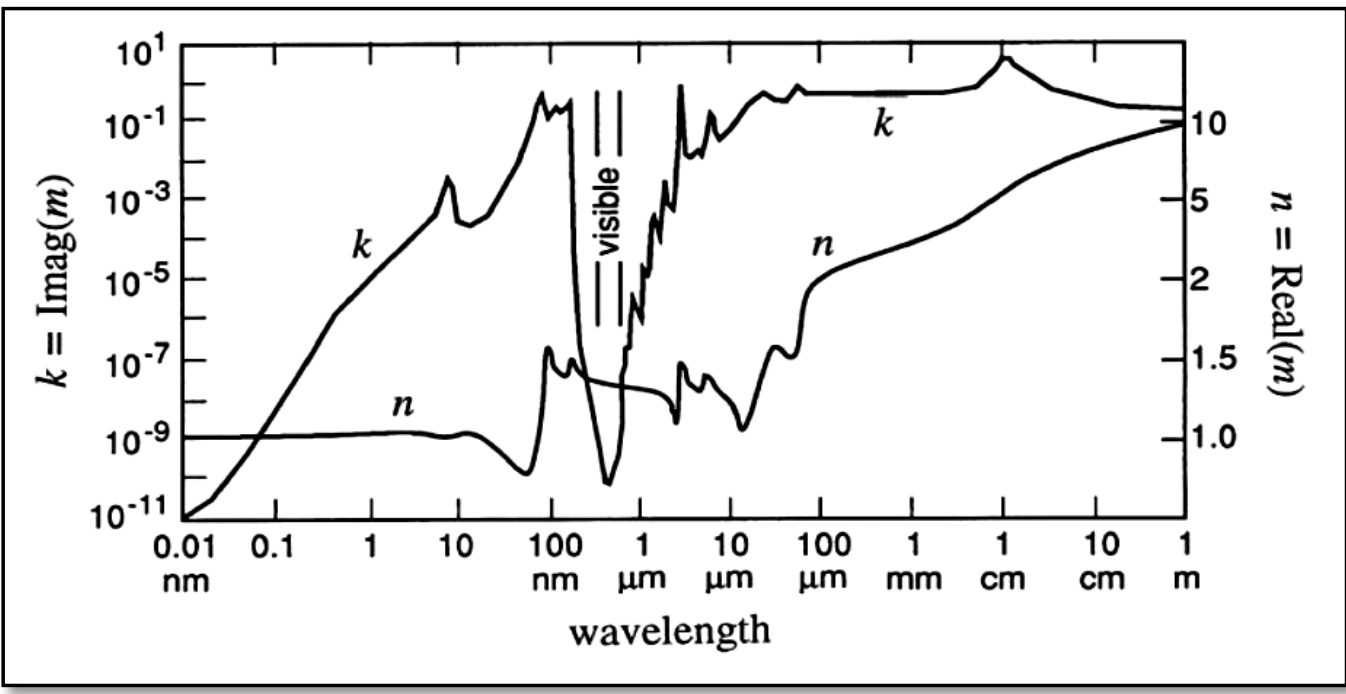

Fig.1. the optical constants of pure water. The left axis gives the imaginary part of $\mathbf{m}$, and the right axis gives the real part of $m$, where $m$ is the complex index of refraction. 


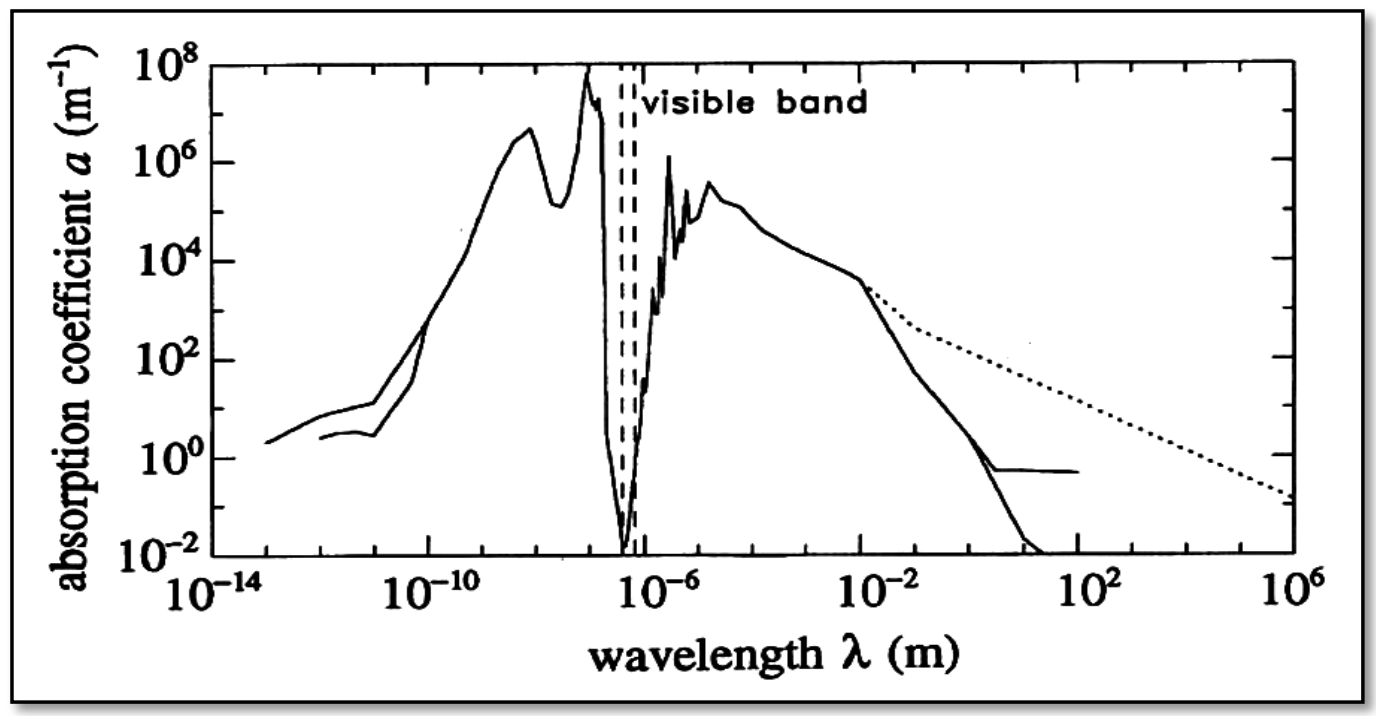

Fig.2 Spectral absorption coefficient of pure water (solid line) and of pure sea water (dotted line) as a function of wavelength.

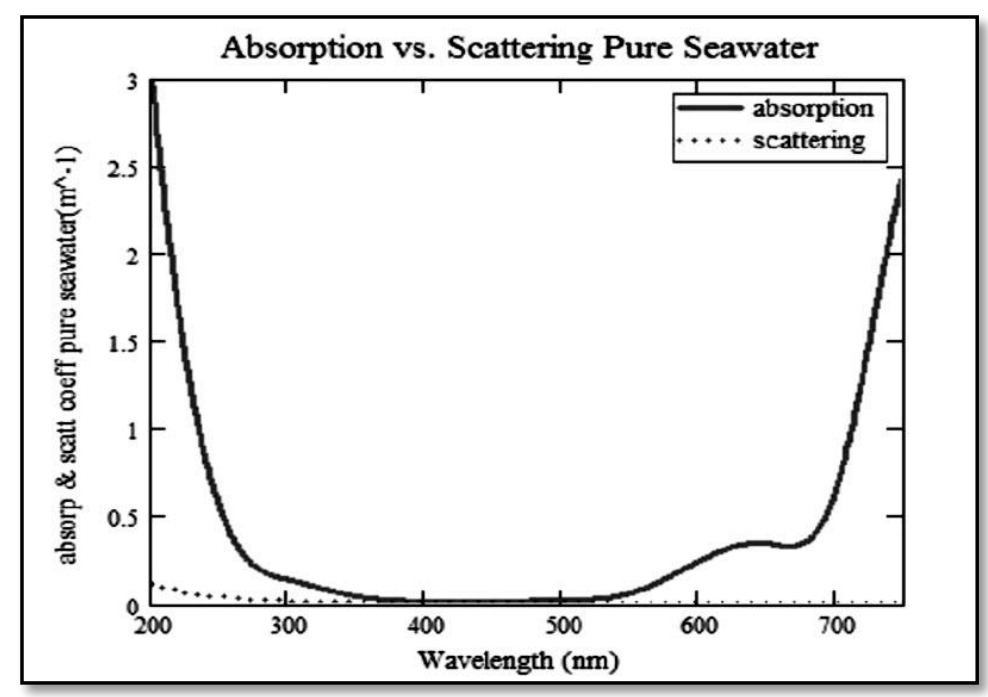

Fig 3 .Absorption and Scattering of water as a function of wavelength $(200: 750 \mathrm{~nm})$.

Fig.1 shows the wavelength dependence of the optical constants $\mathrm{n}$ and $\mathrm{k}$ for pure water. The extraordinary feature seen in this figure is the narrow "window" in $k(\lambda)$, where $k(\lambda)$ decreases by over nine orders of magnitude between the near ultraviolet and the visible, and then quickly rises again in the near infrared. This behavior in $\mathrm{k}(\lambda)$ gives a corresponding window in the spectral absorption coefficient $a(\lambda)$, as seen in Fig.2 the shape of the absorption curve of pure water can be explained as follows. At blue wavelengths, photons are barely energetic enough to boost electrons into higher energy levels of the water molecule, and the photons do not have the right energy to interact 
easily with the molecule as a whole (although some vibrational modes of the molecule can be excited). The photons therefore do not interact strongly with the water molecules, and $\mathrm{a}(\lambda)$ is at its minimum. As the wavelength decreases toward the ultraviolet, the photons become sufficiently energetic to excite atomic transitions, and the absorption rapidly increases. At extremely small wavelengths, processes such as Compton scattering (scattering of high-energy photons by electrons) come in to play, and the relevant parameter is just the density of the material, not its structure as a water molecule. As the wavelength increases from blue to red and beyond, the photons begin having just the right energy to excite first the fundamental vibrational and then the rotational modes of the water molecules, and absorption once again increases rapidly in the infrared. The prominent peaks in $\mathrm{a}(\lambda)$ in the infrared result from these resonant excitations of molecular motions. At very long wavelengths, the photons are not energetic enough to excite molecular motions, and the absorption decreases [7].

Studies on the visible band to investigate the band that has lowest attenuation in water is blue-green light as illustrated in fig.3, due to low absorption and scattering coefficients [8].

Although difficulties of obtaining accurate measurements of the optical absorption of water in the visible and UV regions due to very low values [1], we obtain the absorption coefficient of water in that band using the experimental setup illustrated in fig.4 that consists of LED source (two different sources) with spectrum in fig.5 (a) and fig.5 (b), water sample, a high resolution spectrometer equipped with an integrated sphere. The system used to measure the absorption coefficient of water by comparing the spectrometer spectral intensity of reference LED source after propagating through the water with its corresponding values if propagating through empty glass container at the same distance, the dark spectral intensity was subtracted from both reference and sample intensities; Due to the small variation of absorption coefficient values, it converted to logarithmic scale to distinguish its variation.

The experimental water's absorption coefficient of halogen white LED source in visible band is illustrated in fig.6 and fig.7 illustrate The experimental water's absorption coefficients of the spectrum infra red LED source. It's clear from the absorption coefficient graphs that the blue-green region has the lowest absorption coefficient that provides a high propagation range and suitable for underwater imaging system, but selection of laser source must provide also a high reflection from targets. So, two laser sources (blue and green) were used to distinguish the reflection from different targets (metal, wood and plastic) as illustrated in fig.8 (a) and fig.8 (b), it's clear that the 
reflected laser from metal targets is higher than the plastic and wood targets.the beam profile of the used blue and green laser sources illustrated in fig.9, the Gaussian beam shape of the green laser source provide the precedence to be used for the underwater imaging system.

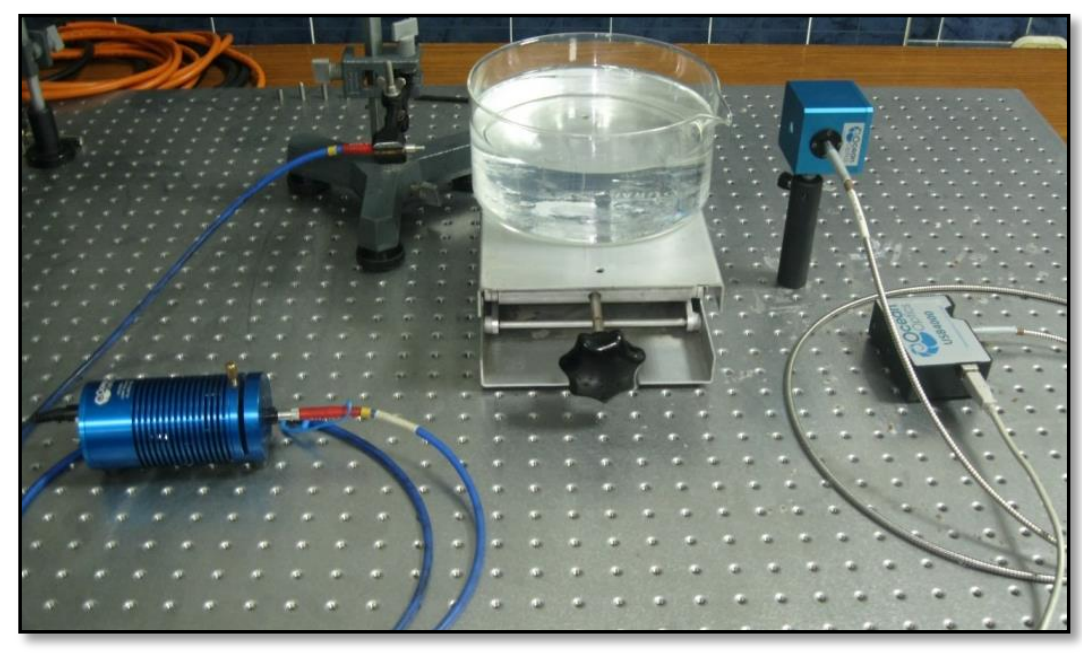

Fig.4 Eprimental setup consisting of LED source, water sample, a high resolution spectrometer equipped with an integrated sphere.

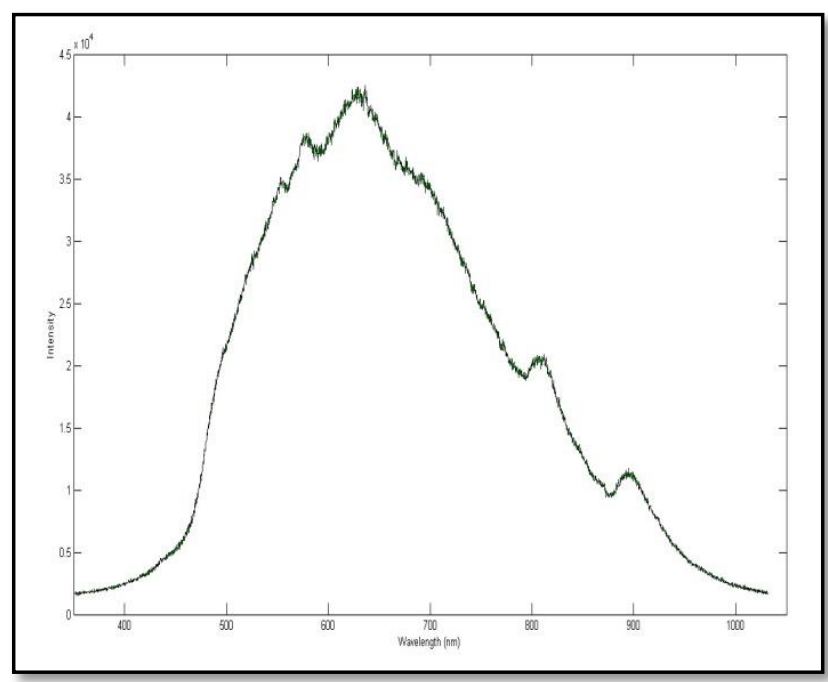

(a)

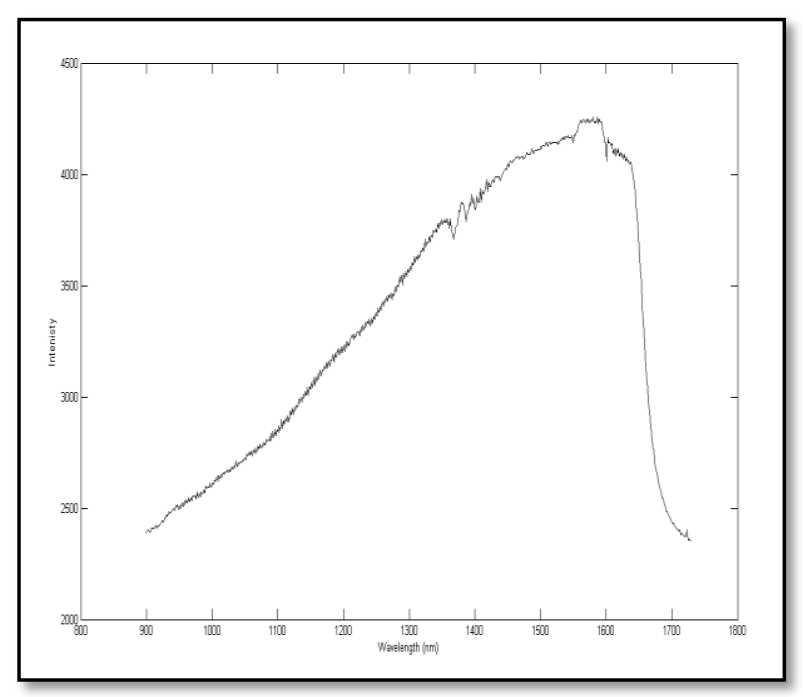

(b)

Fig.5(a) the spectrum Halogen white LED source in visible band, Fig.5(b) the spectrum infra red LED source 


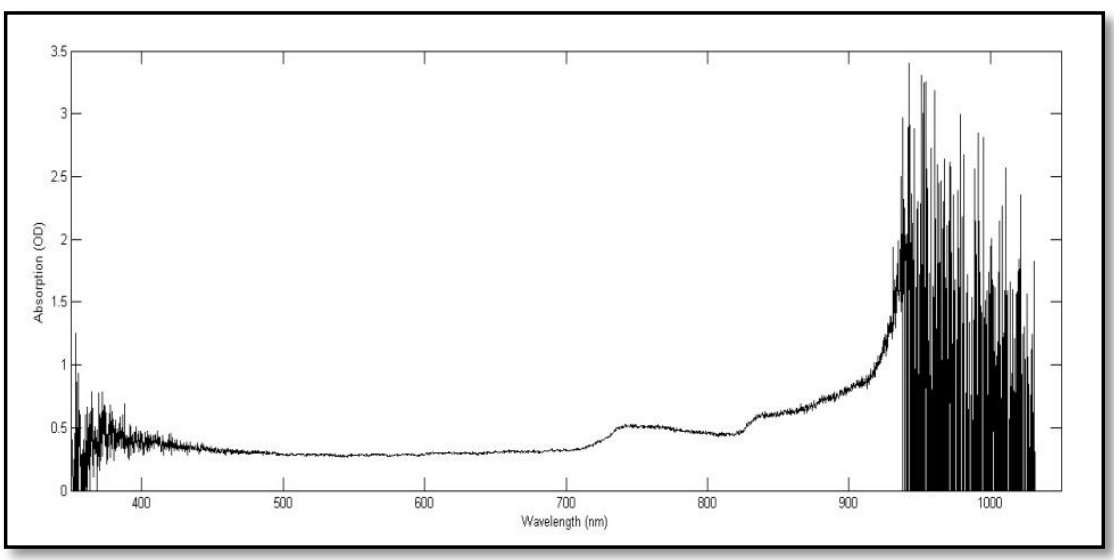

Fig.6 The experimental water's absorption coefficient of Halogen white LED source.

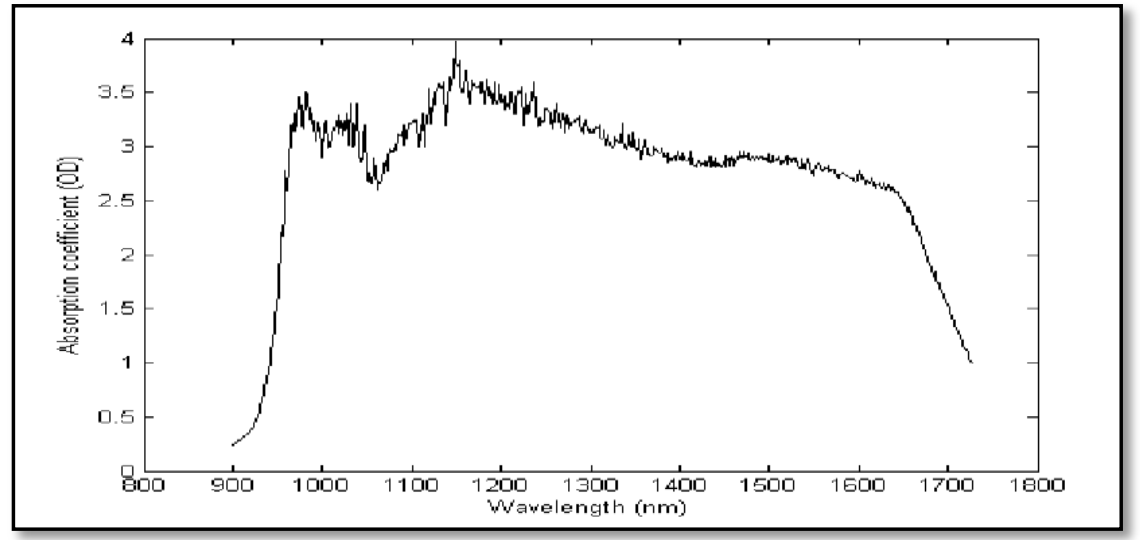

Fig.7 The experimental water's absorption coefficient of the infrared LED source.

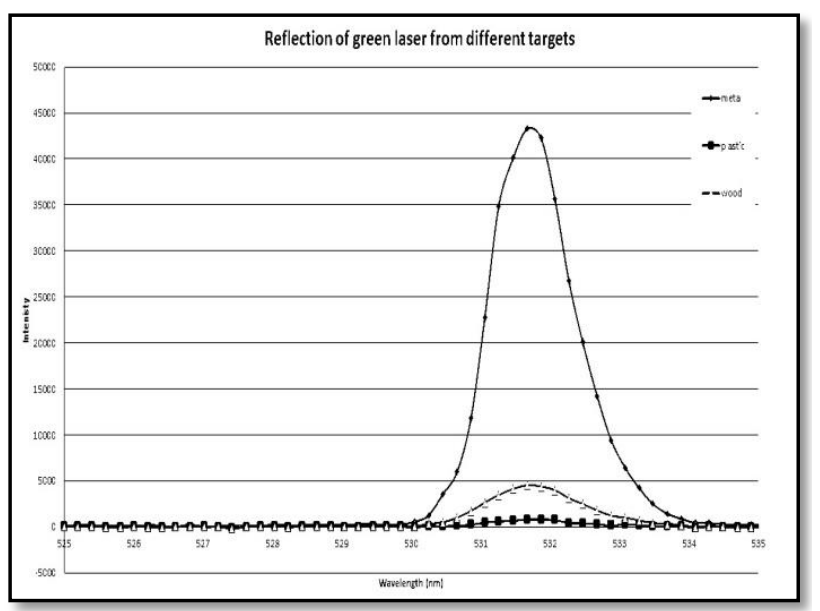

(a)

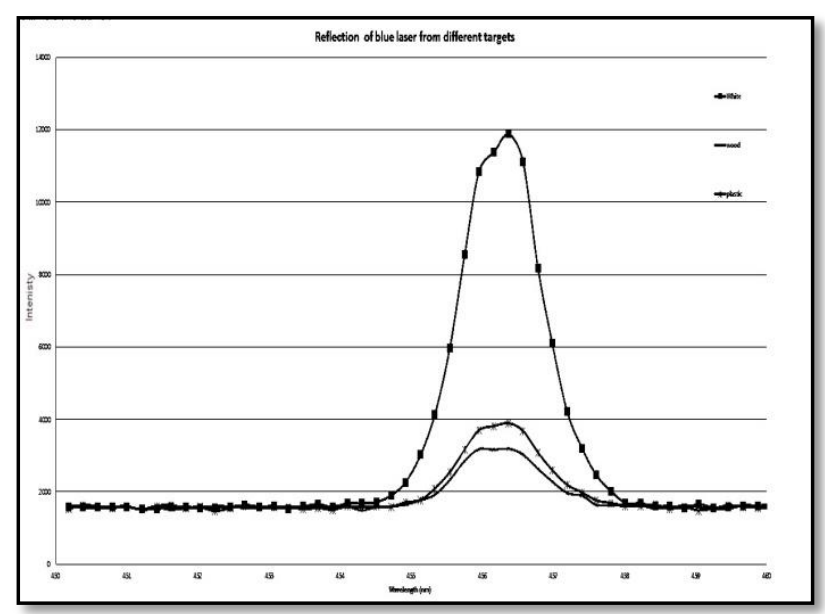

(b)

Fig.8(a) the spectrum Halogen white LED source in visible band, Fig.8(b) the spectrum infra red LED source 


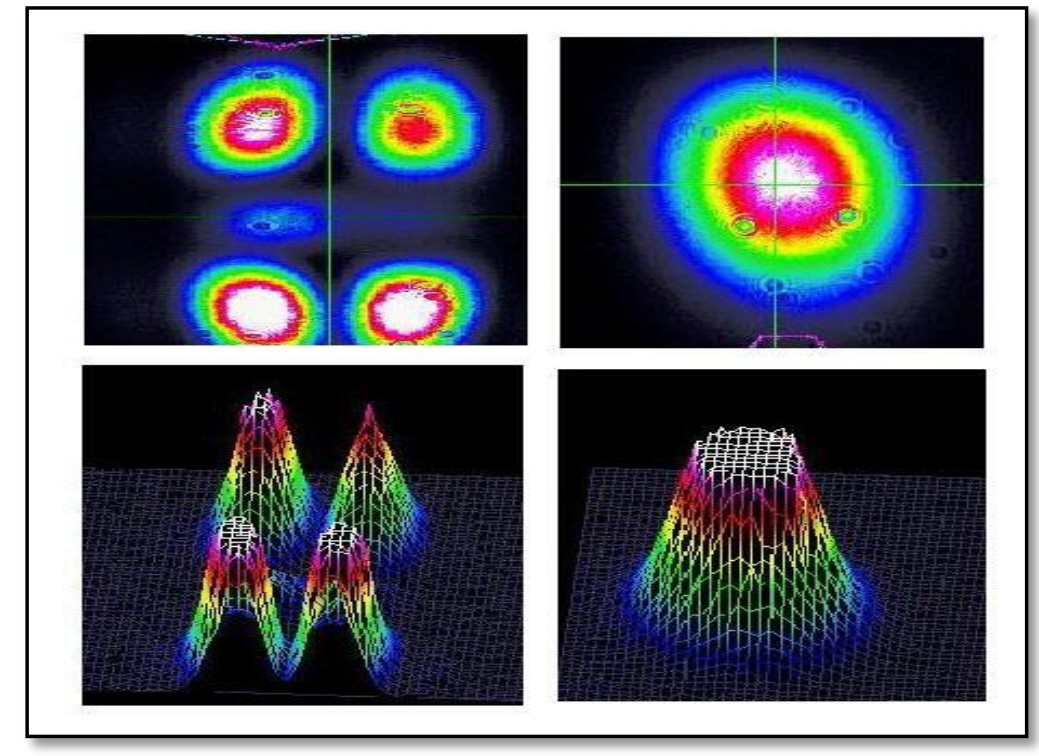

Fig.9 beam profile of blue laser source and the Gaussian beam shape of the green laser source

\section{IMAGING SYSTEM:}

The concept of an underwater laser imaging system was initially proposed in the early 1970's. And the first airborne underwater laser imaging system was used for detecting mines in the period of the second Gulf War and its maximum detection depth can reach to $30 \mathrm{~m}$ under the water. Especially in the last decade, the airborne underwater laser imaging systems has been wildly used in fish detection, search and rescue, oceanic mineral explorations, and oceanic research because of its flexibility and high efficiency [9].

Applications requiring improved image quality and visual information, as well as military surveillance, identification, and detection. Although conventional cameras, including disparity stereo imaging systems, can provide a means for visualization of the work environment, they are limited by poor visibility. With regard to the ideal monochromatic laser and its intense energy, as well as the transparent-windows effect for blue-green light propagating through water, the development of underwater target laser imaging technology has been dramatically increased. The imaging range of target laser imaging system is from tens to over one hundred meters.

When using conventional illumination systems, the amount of backscatter depends on the volume of water where the light field and camera's field of view intersect. Because of this, it is common to separate the light source as much as possible from the camera in 
order to reduce the volume of water. Fig. 10(a) exemplifies the volume of water producing backscatter when the camera and the light source are close, and Fig. 10(b) show the difference when increasing this distance [10].

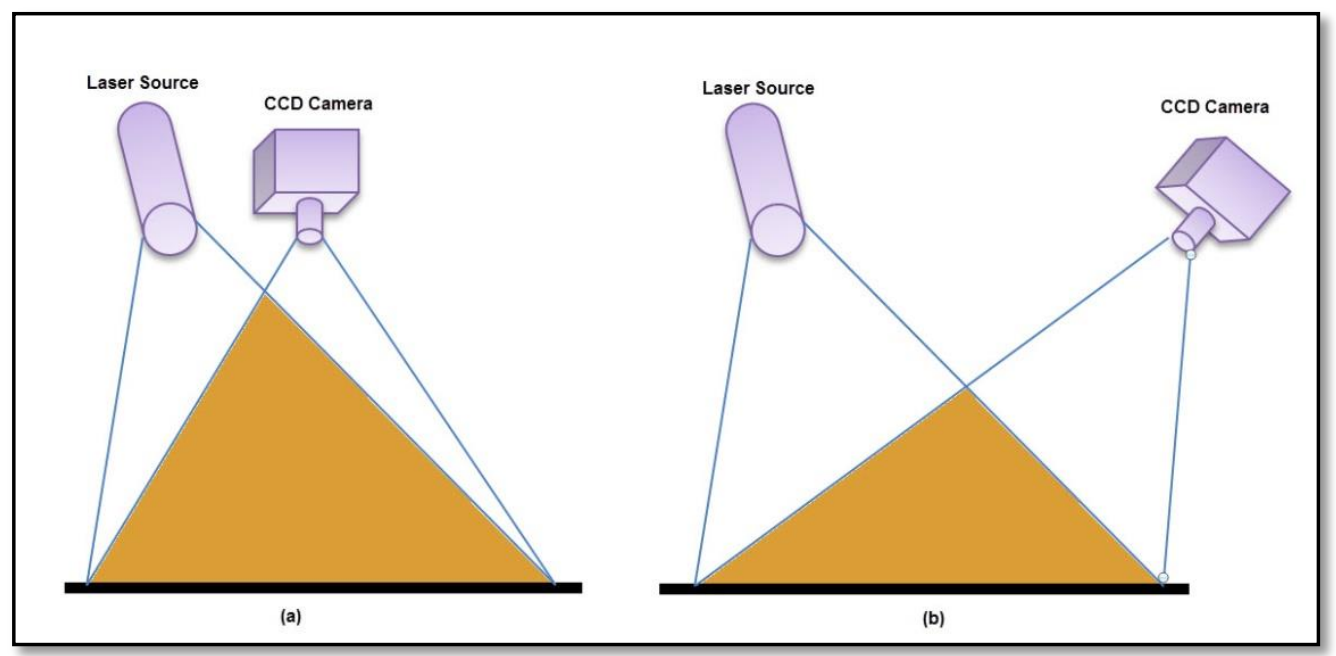

Fig. 10(a) the volume of water producing backscatter when the camera and the light source are close, and Fig. 10(b) show volume of water producing backscatter when increasing this distance.

The underwater laser imaging use the green laser as transmitter via $15 \mathrm{X}$ beam expander to scan the field-of-view, and the receiver (CCD camera) to extract and store reflected signal from target. Then these signals processed by computer to extract image of object. Commonly transmitter device and receiver of underwater laser imaging system was designed as the asymmetry in bi-static configuration to make the illuminating light and return light forming a stated angle to reduce the backscattering and stray light noise as illustrated in the Fig.11, the images captured by CCD camera were applied to simple image processing processes, noise background first is subtracted from the captured image and then reduce the probability of false target alarm by comparing the target image with dynamic threshold and normalize the detected target to increase its contrast in the image, figures from fig. 12 to fig.16 illustrate (a) target image, (b) background image, (c) subtracted image, (d) the normalized image, (e) mesh figure to target to illustrate the intensity level of each pixel of image, (the contour image to determine the position of target in the image, (h) the mesh figure for the target after normalization of the level higher than the dynamic threshold, and (i) the contour figure for the normalized targets, for the target image capture at angle 10,30,50,70, 90 degrees 


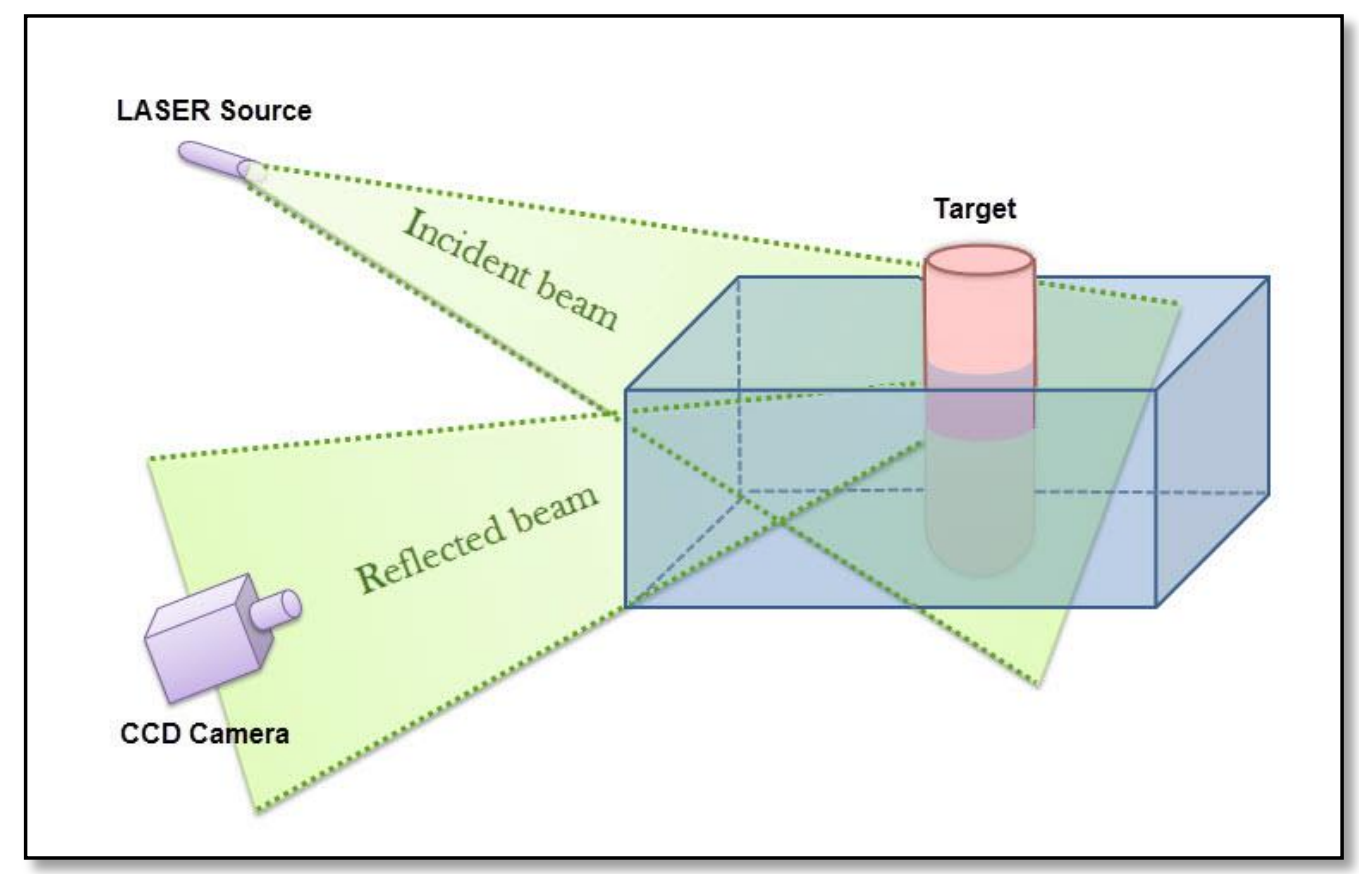

Fig.11 Exprimental imaging system setup consisting of green laser source, immersed target in water, $\mathrm{CCd}$ camera.

(a) Target Image

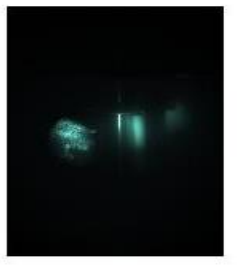

(b) Background Image

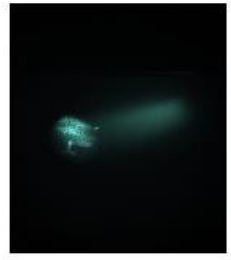

(c) subtracted Image

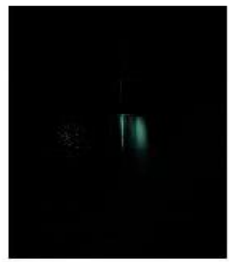

(d) target normalized Image

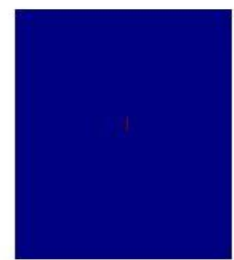

(e) target mesh

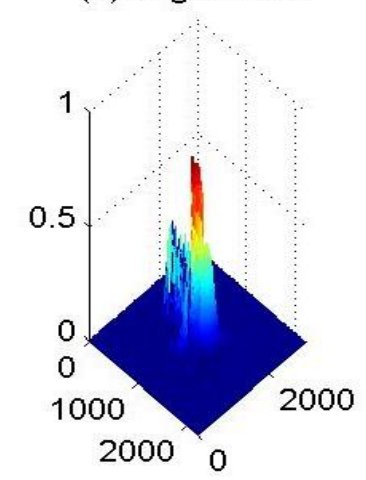

(f) target contour

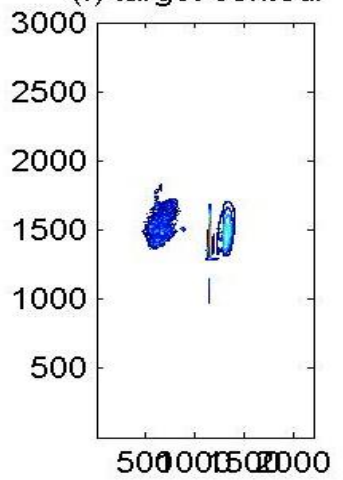

(h) target normalized mes(h) target normalized contour

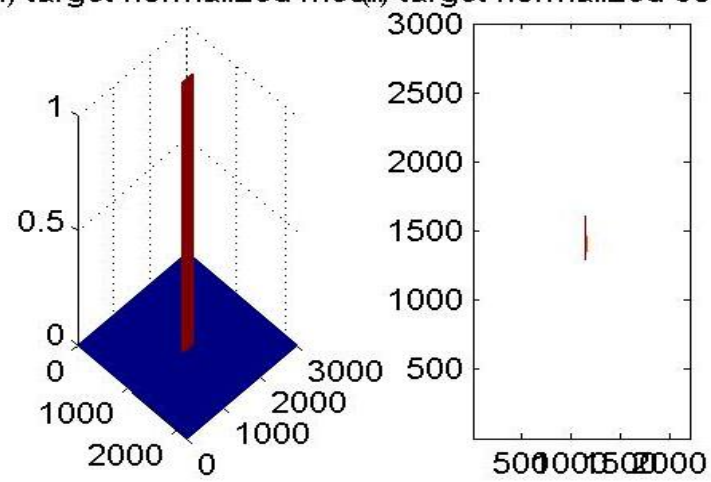

Fig.12 target images captured at 10 degree. 
(a) Target Image

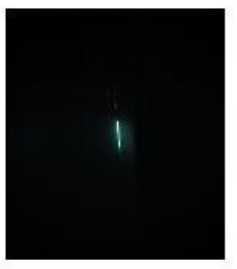

(b) Background Image

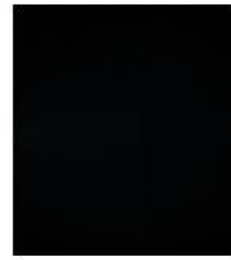

(c) subtracted Image

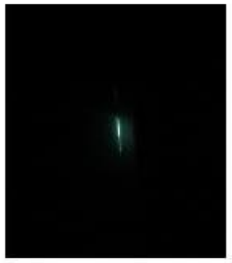

(d) target normalized Image

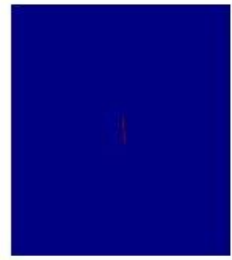

(e) target mesh

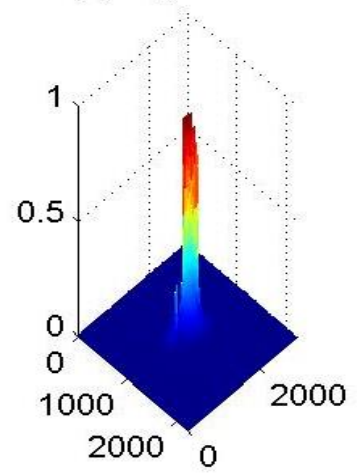

(f) target contour

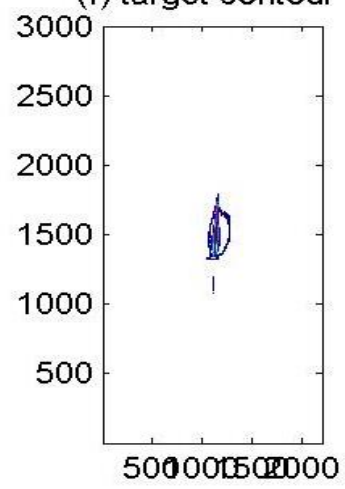

(h) target normalized mes(in) target normalized contour

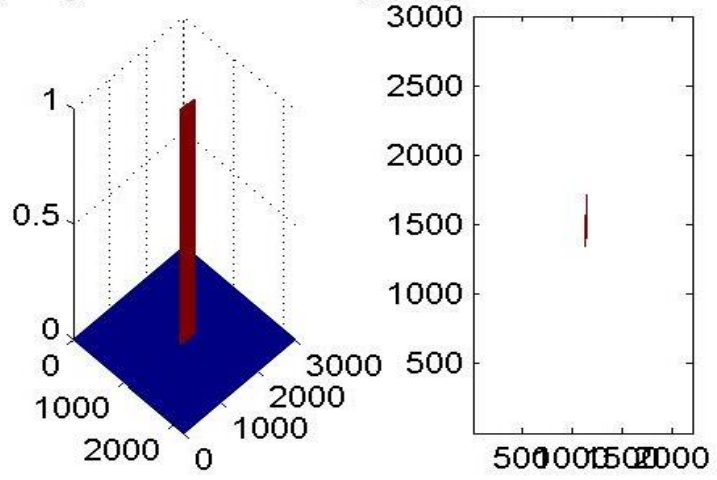

Fig.13 target images captured at 30 degree.

(a) Target Image

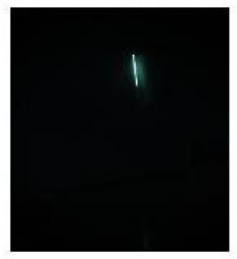

(b) Background Image

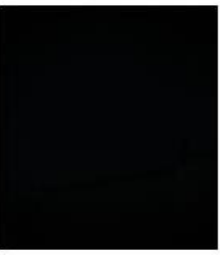

(c) subtracted Image

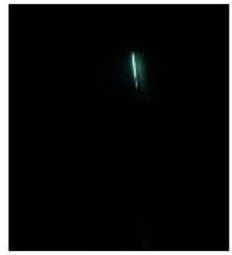

(d) target normalized Image

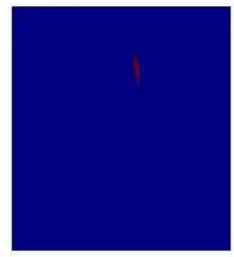

(e) target mesh

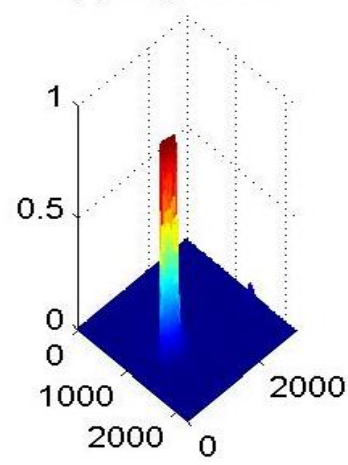

(f) target contour

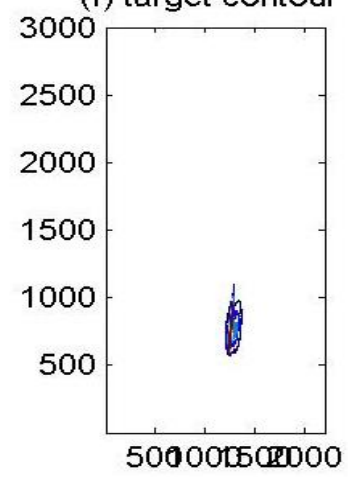

(h) target normalized mes(in) target normalized contour

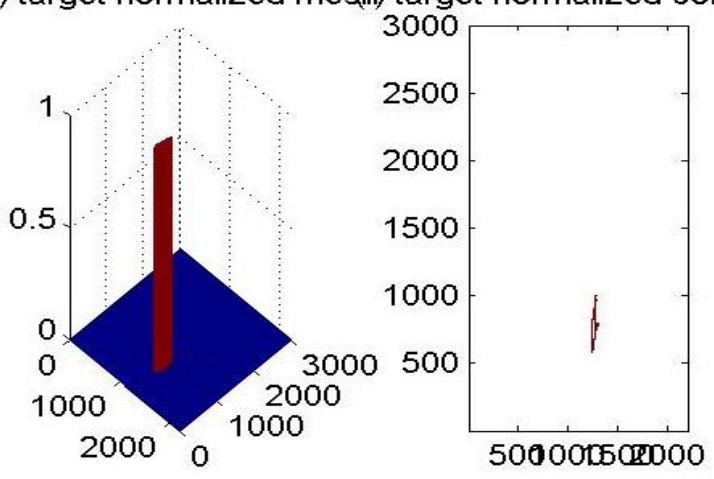

Fig.14 target images captured at 50 degree. 


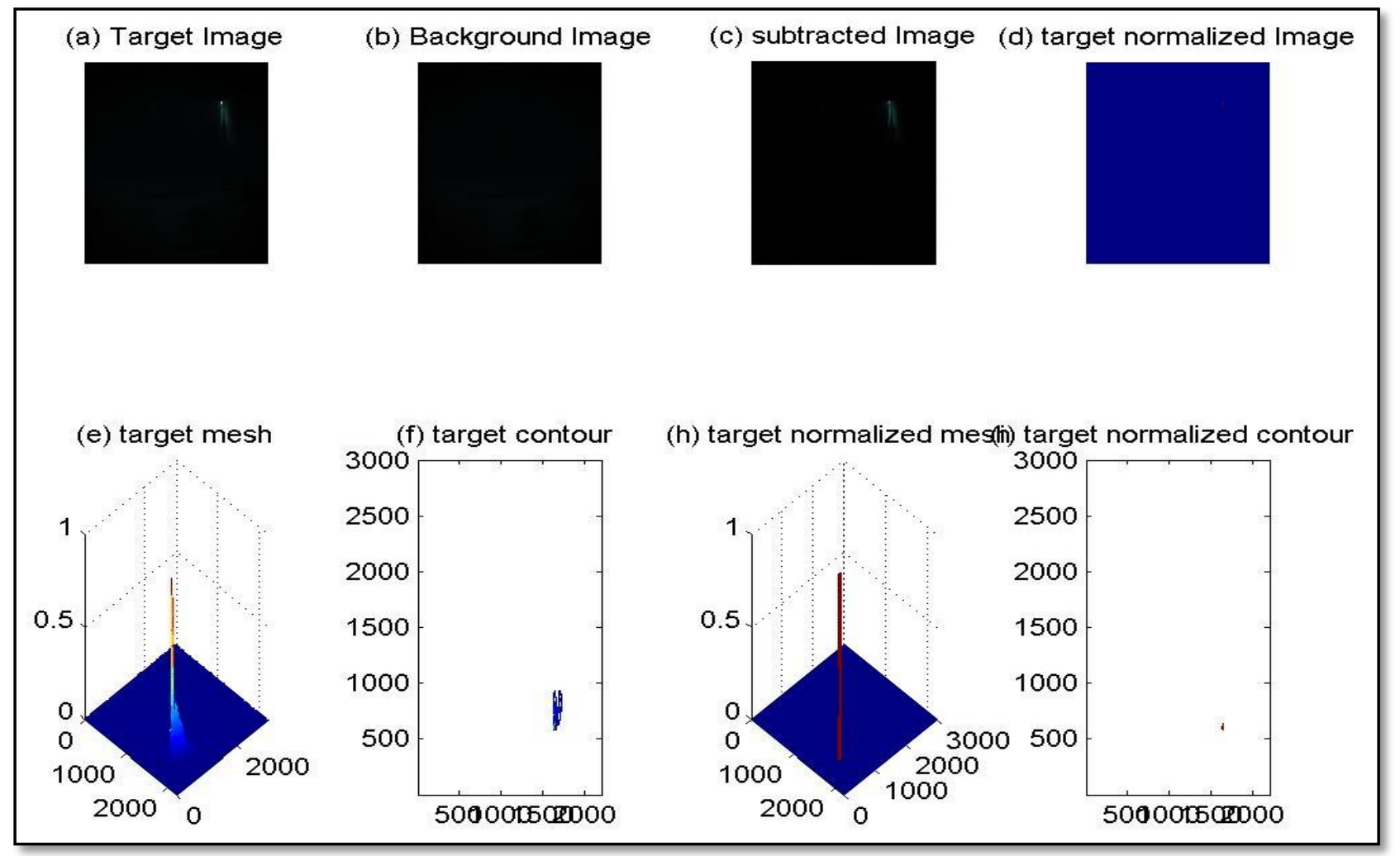

Fig.15 target images captured at 70 degree.

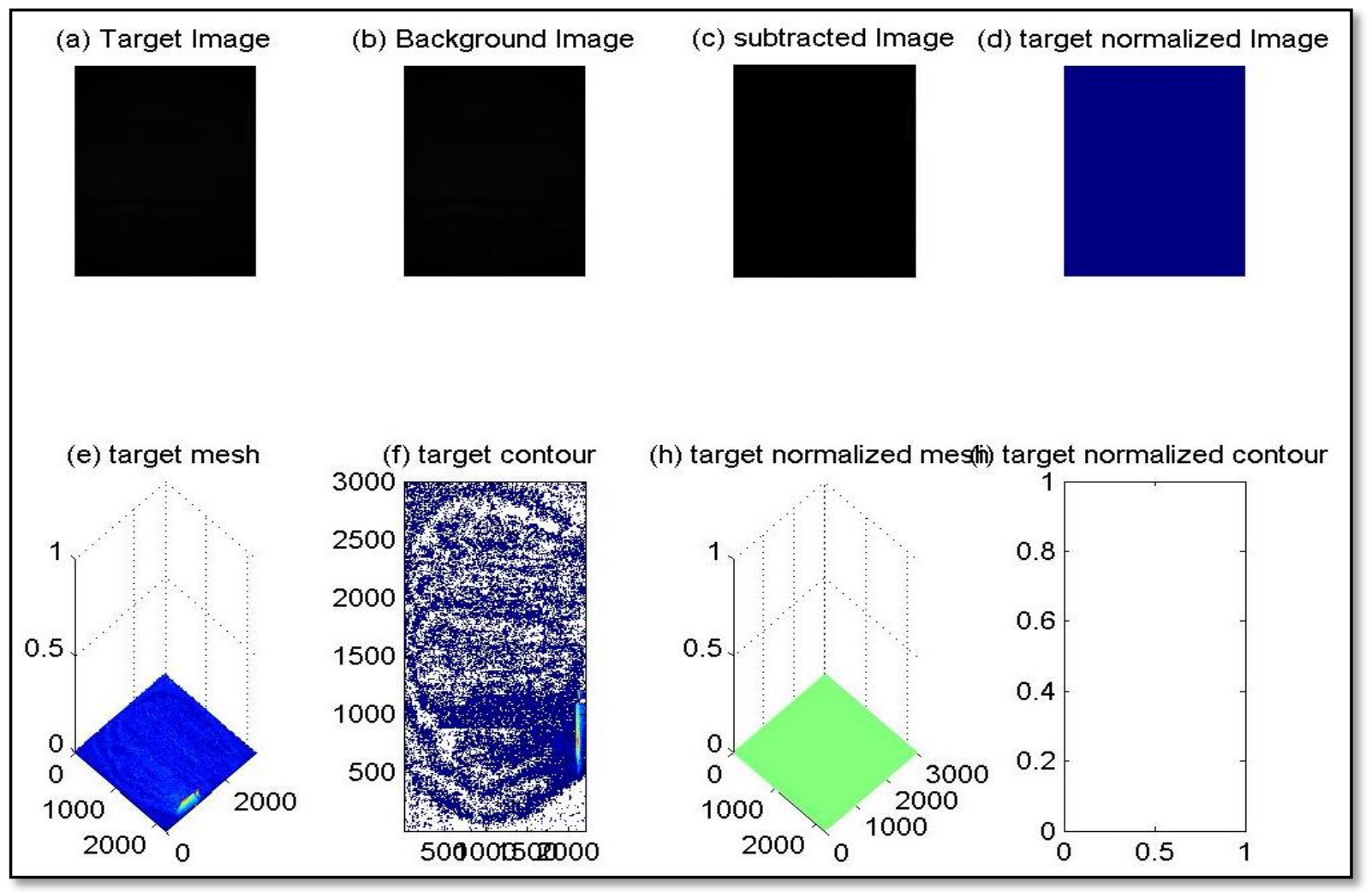

Fig.16 target images captured at 90 degree. 
It's clear from the image at 10 degree that the highest backscattered noise occur due to the distance between the camera and the laser source are close, and this backscattering noise decrease by increasing the distance between them, The optimum distance between the laser source and the camera is corresponding to the angles from 30 to 50 degree for short distances of few meters.

\section{CONCULSION:}

The potential benefits of a laser-based underwater target detection are highdirectionality, high response, and high precise range accuracy. Lasers operating in the blue-green region of the light spectrum have potential applications in the underwater detection and imaging of submersible targets due to minimum attenuation through water and maximum laser reflection of estimated target to provide a long range of detection. in this paper we introduce the parameters of underwater laser imaging system by using blue-green laser as transmitter via beam expander to scan the field-of-view, and the receiver (CCD camera) to extract and store reflected signal from target. Then these signals processed by computer to extract image of object. Commonly blue-green laser source and CCD camera of underwater laser imaging system was designed as the asymmetry in bi-static configuration to make the illuminating light and return light forming a stated angle to reduce the backscattering and stray light noise, the images captured by CCD camera were applied to simple image processing processes to reduce the scattering noise and detect the shape of underwater target.

\section{REFERENCES}

[1] Alan, Laux, Linda Mullen, Paul Perez, and Eleonora, "Under laser range finder", Proc. Of SPIE Vol. 8372, 83721B (2012).

[2] Kilpelä, Ari, "Pulsed time-of-flight laser range finder techniques for fast, high precision measurement applications", Department of Electrical and Information Engineering, University of Oulu- Finland, P.O.Box 4500, FIN-90014 (2004)

[3] Amann, M., Bosch, T., Lescure, M., Myllylä, R., and Rioux M., "Laser ranging: a critical review of usual techniques for distance measurement", Opt. Eng. 40, 10 (2001).

[4] W. Scott Pegau, Deric Gray, and J. Ronald "Absorption and attenuation of visible and near infrared light in water: dependence on temperature and salinity", Applied optics Vol. 36, No. 24 (1997). 
[5] Ling Wang, "Measuring optical absorption coefficient of pure water in UV using the integrating cavity absorption meter”, PHD thesis, Peking University, China (2008).

[6] Milton Kerker M., "The scattering of light and other electromagnetic radiation" book, Publisher: Academic press New York.

[7] Michael Bass, "Handbook of Optics: Volume IV - Optical Properties of Materials, Nonlinear Optics, Quantum Optics”, Third Edition.

[8] LV Pei, HE Junhue, Zhou Renkui, and Liu Haiying "The application of underwater optics development", Proc. Of SPIE Vol. 6837, 68370I, (2007).

[9] Wang Lei, Xu Zhi-yong, Zhang Qi-heng, Wang Hua-chuang, Yu Xue-gang, Nie Rui-jie, "Exprimental underwater scanning imaging system using pulsed blue-green lasers", Proc. of SPIE Vol. 8192 81921K-1, (2011).

[10] F. Bonin, A.Burguera, and G. Olivera, “ Imaging systems for advanced underwater vehicles”, Journal of Maritime research, Vol VIII, No.1, pp.65-86, (2011). 